\title{
Article \\ Clinical Impact of Revised Ciprofloxacin Breakpoint in Patients with Urinary Tract Infections by Enterobacteriaceae
}

\author{
Ga Eun Park ${ }^{1}$, Jae-Hoon $\mathrm{Ko}^{2}$, Sun Young Cho ${ }^{2}$, Hee Jae Huh ${ }^{2}$, Jin Yang Baek ${ }^{3}{ }^{\circledR}$, Kwan Soo Ko ${ }^{3,4}{ }^{\circledR}$, \\ Cheol-In Kang ${ }^{2}$, Doo Ryeon Chung ${ }^{2}$ and Kyong Ran Peck ${ }^{2, *(\mathbb{D}}$ \\ 1 Medical Center, Division of Infectious Diseases, Department of Medicine, Konkuk University, \\ Seoul 05030, Korea; kaeun84@gmail.com \\ 2 Samsung Medical Center, Division of Infectious Diseases, Department of Medicine, Sungkyunkwan \\ University School of Medicine, Seoul 06351, Korea; jaehoon.ko@samsung.com (J.-H.K.); \\ sunyoung81.jo@samsung.com (S.Y.C.); heejae.huh@samsung.com (H.J.H.); collacin@gmail.com (C.-I.K.); \\ iddrchung@gmail.com (D.R.C.) \\ 3 Asia Pacific Foundation for Infectious Diseases (APFID), Seoul 06326, Korea; jy34.baek@gmail.com (J.Y.B.); \\ ksko@skku.edu (K.S.K.) \\ 4 Molecular and Cell Biology, Sungkyunkwan University School of Medicine, Seoul 05030, Korea \\ * Correspondence: krpeck@skku.edu; Tel.: +82-2-3410-0329; Fax: +82-2-3410-0064
}

\section{check for} updates

Citation: Park, G.E.; Ko, J.-H.; Cho, S.Y.; Huh, H.J.; Baek, J.Y.; Ko, K.S.; Kang, C.-I.; Chung, D.R.; Peck, K.R. Clinical Impact of Revised Ciprofloxacin Breakpoint in Patients with Urinary Tract Infections by Enterobacteriaceae. Antibiotics 2021, 10, 469. https://doi.org/10.3390/ antibiotics 10040469

Academic Editors: Catherine Neuwirth and Jeong Seok-Hoon

Received: 12 March 2021

Accepted: 1 April 2021

Published: 20 April 2021

Publisher's Note: MDPI stays neutral with regard to jurisdictional claims in published maps and institutional affiliations.

Copyright: (c) 2021 by the authors. Licensee MDPI, Basel, Switzerland. This article is an open access article distributed under the terms and conditions of the Creative Commons Attribution (CC BY) license (https:/ / creativecommons.org/licenses/by/ $4.0 /)$.

\begin{abstract}
In 2018, the Clinical and Laboratory Standards Institute (CLSI) revised ciprofloxacin (CIP)-susceptible breakpoint for Enterobacteriaceae from $\leq 1 \mu \mathrm{g} / \mathrm{mL}$ to $\leq 0.25 \mu \mathrm{g} / \mathrm{mL}$, based on pharmacokinetic-pharmacodynamic (PK-PD) analysis. However, clinical data supporting the lowered CIP breakpoint are insufficient. This retrospective cohort study evaluated the clinical outcomes of patients with bacteremic urinary tract infections (UTIs) caused by Enterobacteriaceae, which were previously CIP-susceptible and changed to non-susceptible. Bacteremic UTIs caused by Enterobacteriaceae with CIP minimal inhibitory concentration (MIC) $\leq 1 \mu \mathrm{g} / \mathrm{mL}$ were screened, and then patients treated with CIP as a definitive treatment were finally included. Patients in CIP-non-susceptible group ( $\mathrm{MIC}=0.5$ or $1 \mu \mathrm{g} / \mathrm{mL}$ ) were compared with patients in CIP-susceptible group (MIC $\leq 0.25 \mu \mathrm{g} / \mathrm{mL}$ ). Primary endpoints were recurrence of UTIs within 4 weeks and 90 days. A total of 334 patients were evaluated, including 282 of CIP-susceptible and 52 of CIP-non-susceptible. There were no significant differences in clinical outcomes between two groups. In multivariate analysis, CIP non-susceptibility was not associated with recurrence of UTIs. CIP non-susceptibility based on a revised CIP breakpoint, which was formerly susceptible, was not associated with poor clinical outcomes in bacteremic UTI patients were treated with CIP, similar to those of the susceptible group. Further evaluation is needed to guide the selection of definitive antibiotics for UTIs.
\end{abstract}

Keywords: Enterobacteriaceae; ciprofloxacin; urinary tract infections

\section{Introduction}

Urinary tract infections (UTIs) are the most commonly encountered bacterial infection in the community [1,2]. In hospitals, UTIs are the second most common infection, accounting for nearly $25 \%$ of all infections [3]. Fluoroquinolones are one of most frequently prescribed classes of antibiotics in outpatient and inpatient settings. They have bactericidal action resulting from inhibition of topoisomerase II (DNA gyrase) and topoisomerase IV, which contributes to DNA replication, repair, and recombination. Ciprofloxacin (CIP) is a member of the fluoroquinolones group, which showed in vitro activity against both Gram negative bacilli and Gram positive bacteria, including Enterobacteriaceae [4]. CIP is one of the antibiotics most frequently prescribed for treating UTIs. This is due to the fact that CIP has a good bactericidal effect, clinical cure rates, high bioavailability exceeding $70 \%$, and high concentrations in urine and kidney tissue [5]. However, resistance to quinolone has emerged all over the world following their widespread use. Three mechanisms of 
quinolone resistance have been reported: (1) chromosomal mutations in the genes encoding quinolone target enzymes (i.e., DNA gyrase or topoisomerase IV) that occur most often in a region referred to as the quinolone resistance determining region (QRDR); (2) the acquisition of plasmid-mediated quinolone resistance (PMQR) genes; and (3) chromosomal mutations that decrease intracellular concentration of quinolone by modification of the efflux pumps [6,7]. Recently, the Clinical and Laboratory Standards Institute (CLSI) revised CIP-susceptible breakpoint for Enterobacteriaceae from $\leq 1 \mu \mathrm{g} / \mathrm{mL}$ to $\leq 0.25 \mu \mathrm{g} / \mathrm{mL}$, based on pharmacokinetic-pharmacodynamic (PK-PD) attainment analyses and in vitro bacteria eradication data [8-12]. However, PK-PD analysis and in vitro data may not accurately predict clinical outcomes for all infections, especially less severe infections such as UTIs. Moreover, clinical data supporting the lowered CLSI breakpoint are insufficient, and this revised MIC is also not based on the mechanism of quinolone resistance. In order to evaluate the clinical impact of revised CLSI breakpoint, we analyzed the clinical outcomes of patients receiving CIP for the treatment of UTIs with bacteremia caused by Enterobacteriaceae isolates that were previously CIP-susceptible and changed to non-susceptible. We compared those with CIP minimal inhibitory concentration (MIC) $\leq 0.25 \mathrm{~g} / \mathrm{mL}$ and those with CIP MIC 0.5 and $1 \mathrm{~g} / \mathrm{mL}$.

\section{Results}

\subsection{Study Populations, Baseline Characteristics, and Severity of Infections}

A total of 334 patients were eligible. Fifty-two patients were in CIP-susceptible group and 282 patients were in CIP-non-susceptible group (Figure 1). Demographic characteristics are shown in Table 1 . There were no significant differences between the two groups with regard to age, Charlson's weighted index of comorbidity (CWIs), identified pathogens including extended-spectrum beta-lactamases (ESBL) producing organisms, treatment duration of antibiotics, and number of risk factors for recurrent UTIs. The most frequent etiologic organism was Escherichia coli (91\%). CIP was empirically used as initial treatment regimen in 33\% of 334 patients. The third generation cephalosporin (ceftriaxone or cefotaxime) was used in $40.4 \%$. The proportion of patients receiving CIP as an empirical as well as a definitive treatment did not differ between CIP-susceptible and CIP-non-susceptible group (33\% vs. 34.6\%, $p=0.818$, respectively). Female patients were more common in CIP-non-susceptible group than CIP-susceptible group (94.2\% vs. $80.5 \%, p=0.016$, respectively). A lower proportion of patients in the CIP-susceptible group had liver disease $(9.6 \%$ vs. $19.2 \%, p=0.041$, respectively) and renal disease ( $4.3 \%$ vs. $11.5 \%$. $p=0.033$, respectively) compared to patients in the CIP-non-susceptible group, and a higher proportion of patients in the CIP-susceptible group had solid cancer $(16.3 \%$ vs. $5.8 \%, p=0.044$, respectively). The median value of pitt bacteremia score was not different between CIP-susceptible group and CIP-non-susceptible group, whereas standard deviation of median value was statistically significantly higher in CIP-susceptible group (1 [0-2] vs. 1 [0,1], $p=0.006$, respectively). The proportion of patients with risk factors for recurrence of UTIs did not differ between two groups $(26.6 \%$ vs. $26.9 \%, p=0.961)$

\subsection{Clinical Outcomes of Patients with Enterobacteriaceae Bacteremic UTIs}

The clinical outcomes of patients receiving CIP for the treatment of UTIs with Enterobacteriaceae bacteremia are presented in Table 2. Patients in the CIP-susceptible group remained hospitalized longer than patients in the CIP-non-susceptible group, but a statistically significant difference was not observed (6 (4-8) vs. 5 (4-6.75) days, $p=0.055)$. There were no significant differences in recurrence within 4 weeks $(2.5 \%$ vs. $3.8 \%, p=0.577$, respectively), recurrence within 90 -days ( $8.2 \%$ vs. $5.8 \%, p=0.399$, respectively), and all-cause mortality ( $1.1 \%$ vs. $1.9 \%, p=0.494$, respectively) between CIP-susceptible and CIP-nonsusceptible group. Out of 334 patients, 282 patients underwent follow-up blood culture, and prolonged bacteremia more than one week were not observed in either group. Time to defervescence was longer in CIP-susceptible group than CIP-non-susceptible group (3 (2-4) vs. 2 (1-3) days, $p=0.003$, respectively). 


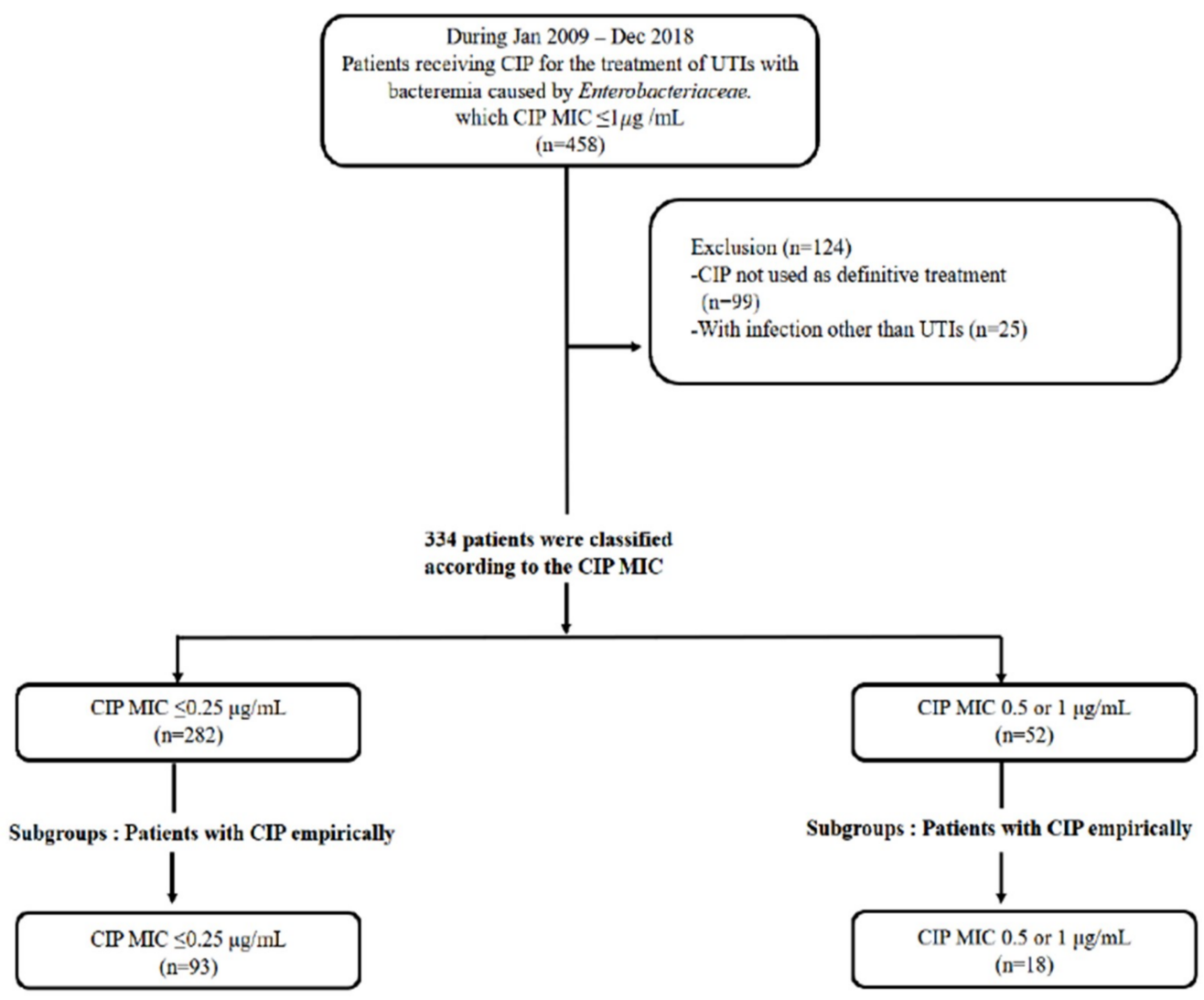

Figure 1. Flowchart of study population.

Table 1. Baseline characteristics of patients with bacteremic urinary tract infections caused by Enterobacteriaceae in ciprofloxacin-susceptible group and ciprofloxacin-non-susceptible group.

\begin{tabular}{|c|c|c|c|}
\hline Variables & $\begin{array}{l}\text { CIP-Susceptible } \\
\text { MIC } \leq 0.25 \\
(n=282)\end{array}$ & $\begin{array}{c}\text { CIP-Non-Susceptible } \\
\text { MIC }=0.5 \text { or } 1 \\
(n=52)\end{array}$ & $p$ Value \\
\hline Sex, female & $227(80.5)$ & $49(94.2)$ & 0.016 \\
\hline Age (years) & $69(55-78)$ & $68.5(51-77)$ & 0.363 \\
\hline \multicolumn{4}{|l|}{ Patients with any comorbidity } \\
\hline Diabetes mellitus & $79(28)$ & $15(28.8)$ & 0.902 \\
\hline Cardiovascular disease & $113(40.1)$ & $18(34.6)$ & 0.459 \\
\hline Respiratory disease & $6(2.1)$ & $0(0)$ & 0.359 \\
\hline Liver disease & $27(9.6)$ & $10(19.2)$ & 0.041 \\
\hline Renal disease & $12(4.3)$ & $6(11.5)$ & 0.033 \\
\hline Neurologic disease & $34(12.1)$ & $6(11.5)$ & 0.916 \\
\hline Solid cancer & $46(16.3)$ & $3(5.8)$ & 0.048 \\
\hline Connective tissue disease & $10(3.5)$ & $2(3.8)$ & 0.915 \\
\hline Hematologic disease & $4(1.4)$ & $1(1.9)$ & 0.573 \\
\hline Solid organ transplantation & $15(5.3)$ & $6(11.5)$ & 0.090 \\
\hline CWI score & $1(0-2)$ & $1(0-2)$ & 0.558 \\
\hline Pitt bacteremia score & $1(0-2)$ & $1(0-1)$ & 0.006 \\
\hline \multicolumn{4}{|l|}{ Identified pathogen } \\
\hline Escherichia coli & $256(90.8)$ & $48(92.3)$ & 0.723 \\
\hline Klebsiella pneumoniae & $26(9.2)$ & $4(7.7)$ & 0.723 \\
\hline $\begin{array}{c}\text { ESBL } \\
\text { Producing organism }\end{array}$ & $10(3.5)$ & $2(3.8)$ & 0.915 \\
\hline \multicolumn{4}{|l|}{ Empirical antibiotics } \\
\hline Appropriate antibiotics & $278(98.6)$ & $33(63.5)$ & $<0.001$ \\
\hline Ciprofloxacin & $93(33)$ & $18(34.6)$ & 0.818 \\
\hline Ceftriaxone & $114(40.3)$ & $21(40.4)$ & 0.996 \\
\hline
\end{tabular}


Table 1. Cont.

\begin{tabular}{|c|c|c|c|}
\hline Variables & $\begin{array}{c}\text { CIP-Susceptible } \\
\text { MIC } \leq 0.25 \\
(n=282)\end{array}$ & $\begin{array}{c}\text { CIP-Non-Susceptible } \\
\text { MIC }=0.5 \text { or } 1 \\
(n=52)\end{array}$ & $p$ Value \\
\hline Piperacillin/tazobactam & $47(16.7)$ & $9(17.3)$ & 0.909 \\
\hline Ertapenem & $5(1.8)$ & $1(1.9)$ & 0.940 \\
\hline Others & $23(8.2)$ & $3(5.8)$ & 0.399 \\
\hline Treatment duration & $13(11-17)$ & $13(10-15)$ & 0.119 \\
\hline Follow up duration & $107(63-141.50)$ & $100(43.25-131.25)$ & 0.401 \\
\hline Risk factors for recurrence & $75(26.6)$ & $14(26.9)$ & 0.961 \\
\hline Previous UTIs history & $36(12.8)$ & $8(15.4)$ & 0.608 \\
\hline Foreign body insertion & $14(5)$ & $0(0)$ & 0.101 \\
\hline Urinary stone & $12(4.3)$ & $0(0)$ & 0.130 \\
\hline Polycystic kidney disease & $1(0.4)$ & $0(0)$ & 0.844 \\
\hline Urinary dysfunction & $12(4.3)$ & $3(5.8)$ & 0.628 \\
\hline Kidney transplantation & $13(4.6)$ & $6(11.5)$ & 0.047 \\
\hline
\end{tabular}

Data are expressed as number (\%) of patients or median (IQR). Abbreviations: CIP = ciprofloxacin; MIC = minimum inhibitory concentration; $\mathrm{CWI}=$ Charlson weighted index; ESBL = extended-spectrum beta-lactamases; UTIs = urinary tract infections .

Table 2. Comparison of clinical outcomes for patients with bacteremic urinary tract infections with Enterobacteriaceae bacteremia ciprofloxacin-susceptible group and ciprofloxacin-non-susceptible group.

\begin{tabular}{cccc}
\hline Variables & $\begin{array}{c}\text { CIP-Susceptible } \\
\text { MIC } \leq \mathbf{0 . 2 5} \\
(\boldsymbol{n = 2 8 2 )}\end{array}$ & $\begin{array}{c}\text { CIP-Non-Susceptible } \\
\text { MIC }=\mathbf{0 . 5} \text { or } \mathbf{1} \\
(\boldsymbol{n}=\mathbf{5 2})\end{array}$ & $\boldsymbol{p}$ Value \\
\hline Length of Hospital days & $6(4-8)$ & $5(4-6.75)$ & 0.055 \\
Recurrence within 4 weeks & $7(2.5)$ & $2(3.8)$ & 0.577 \\
Recurrence within 90 days & $23(8.2)$ & $3(5.8)$ & 0.399 \\
All-cause mortality & $3(1.1)$ & $1(1.9)$ & 0.494 \\
Prolonged bacteremia more than 1 week & $0(0)$ & $0(0)$ & 1 \\
Time to defervescence & $3(2-4)$ & $2(1-3)$ & 0.003 \\
\hline
\end{tabular}

Data are expressed as number (\%) of patients or median (IQR). Abbreviations: CIP = ciprofloxacin; MIC = minimum inhibitory concentration.

\subsection{Multivariate Analysis of Relapse within 4 weeks and 90 days Recurrence}

There were a few meaningful variables in univariate analysis of association between characteristics of patients and recurrence of UTIs within 4 weeks (Supplementary Table S1) and 90 days (Supplementary Table S2). A multivariate cox regression analysis was performed to adjust for the effects of potential confounding factors on recurrence of UTIs within 4 weeks and 90-days. Meaningful variables in univariate analysis were replaced with variables that could be represented. In addition to CIP non-susceptibility, age, sex, ESBL producing organism, CWIs, appropriate antibiotics, pitt bacteremia score, and risk factors for recurrent UTIs were included in the multivariable analysis. In the multivariate analysis, CIP nonsusceptibility were not associated with recurrence within 4 weeks (Table 3) and 90 days (Table 4). Having a known risk factor for recurrence of UTIs was identified as a significant variable for recurrence of UTIs within 90 days (HR 2.370, 95\% CI 1.050-5.351, $p=0.038$ ). 
Table 3. Multivariate analysis of association between characteristics of patients and recurrence of urinary tract infections within 4 weeks.

\begin{tabular}{ccc}
\hline Variables & HR $(\mathbf{9 5} \% \mathbf{C I})$ & $p$ Value \\
\hline Sex, female & $1.391(0.164-11.827)$ & 0.763 \\
Age & $1.025(0.979-1.073)$ & 0.298 \\
CIP, non-susceptible & $0.888(0.091-8.698)$ & 0.918 \\
ESBL producing organism & $1.488(0.099-22.392)$ & 0.774 \\
CWI score & $0.904(0.585-1.397)$ & 0.649 \\
Appropriate antibiotics & $0.235(0.017-3.240)$ & 0.279 \\
Pitt bacteremia score & $1.267(0.828-1.937)$ & 0.275 \\
Risk factors for recurrence & $0.356(0.044-2.886)$ & 0.334 \\
\hline
\end{tabular}

Abbreviations: CIP = ciprofloxacin; ESBL = extended-spectrum beta-lactamases; CWI = Charlson weighted index

Table 4. Multivariate analysis of association between characteristics of patients and recurrence of urinary tract infections within 90 days.

\begin{tabular}{ccc}
\hline Variables & HR $(\mathbf{9 5} \% \mathbf{C I})$ & $p$ Value \\
\hline Sex, female & $0.910(0.331-2.504)$ & 0.855 \\
Age & $0.979(0.957-1.001)$ & 0.066 \\
CIP, non-susceptible & $0.365(0.075-1.791)$ & 0.214 \\
ESBL producing organism & $1.538(0.251-9.432)$ & 0.642 \\
CWI score & $1.030(0.854-1.241)$ & 0.760 \\
Appropriate antibiotics & $0.316(0.052-1.918)$ & 0.211 \\
Pitt bacteremia score & $0.847(0.578-1.242)$ & 0.395 \\
Risk factors for recurrence & $2.370(1.050-5.351)$ & 0.038 \\
\hline Abbreviations: CIP = ciprofloxacin; ESBL = extended-spectrum beta-lactamases; CWI = Charlson weighted index.
\end{tabular}

\subsection{A Subgroup of 111 Patients Treated with CIP Empirically as Well as Definitively}

We also performed a subgroup analysis of patients empirically treated with CIP in CIP-susceptible group and CIP-non-susceptible group. Baseline characteristics of patients treated with CIP as an empirical treatment in CIP-susceptible group and CIP-nonsusceptible group showed in Supplementary Table S3. Bacteremia due to K.pneumoniae was significantly more common in CIP-non-susceptible group than CIP-susceptible group $(22.2 \%$ vs. $6.5 \%, p=0.032$, respectively). Except for this, there were no significant differences between two groups. Clinical outcomes were also not different between two groups (Supplementary Table S4). Comparison of the two groups was performed in the same methods as the primary analysis. CIP non-susceptibility was not associated with recurrence of UTIs within 4 weeks (HR 3.290, 95\% CI 0.190-57.010, $p=0.413$ ) (Supplementary Table S5) and 90 days (HR 0.945, 95\% CI 0.092-9.682, $p=0.962$ ) (Supplementary Table S6). Only age was associated with recurrence within 90 days (HR 0.926, 95\% CI 0.862-0.994, $p=0.033$ ).

\section{Discussion}

The susceptible breakpoints for antibiotics are essential for the clinicians to select an appropriate antibiotics for many types of infectious diseases. In fact, the work of establishing breakpoints conducted by the CLSI or the European Committee on Antimicrobial Susceptibility Testing (EUCAST) is basically based on PK and PD parameters of antibiotics [10]. Although the Clinical and Laboratory Standards Institute (CLSI) revised CIP-susceptible breakpoint for Enterobacteriaceae from $\leq 1 \mu \mathrm{g} / \mathrm{mL}$ to $\leq 0.25 \mu \mathrm{g} / \mathrm{mL}$ in 2018, clinical impact of the lowered CLSI breakpoint has not been evaluated. This retrospective cohort study showed that the CIP non-susceptibility according to revised CIP breakpoint, which was susceptible according to previous criteria, was not associated with recurrence of UTIs within 4 weeks and 90 days.

While there is growing evidence that inappropriate empirical antibiotic treatment may have a negative impact on clinical outcomes, its impact may also be confounded by other variables. Since Enterobacteriaceae, especially E. coli, is one of the main causative 
pathogens of both community- and hospital-acquired infection, the emergence of quinolone resistance of these pathogens is of great concern [13]. To date, many clinical studies have tried to evaluate the association between inadequate empirical treatment due to quinolone resistance and clinical impact in Enterobacteriaceae infections. Lautenbach et al. showed that quinolone resistance was an independent risk factor for mortality in patients with hospital acquired Enterobacteriaceae infection [13], whereas other studies have not. Peralta et al. showed that inadequate antibiotics were associated with worse prognosis in patients with E. coli bacteremia. However, bacteremia of urinary origin was associated with better prognosis [14]. Another previous report showed that discordant use of fluoroquinolone as an initial empirical treatment for bacteremic UTIs caused by Enterobacteriaceae could lead to worse clinical response and longer hospitalization. However, overall mortality and clinical cure rates were not associated with appropriateness of empirical antibiotics treatment [15]. Jeon J.H. et al. showed that that CIP is an appropriate choice for empirical treatment of UTIs and has no serious adverse outcomes, if it is adjusted appropriately, even for patients infected with CIP-resistant organisms [16]. As various studied had shown, bacteremic UTIs caused by Enterobacteriaceae, in particular, accounts for a large proportion of blood stream infections, which are often transient and have a better prognosis than other site of infections [14-18].

To identify the clinical outcomes according to revised CIP breakpoint in UTIs with Enterobacteriaceae bacteremia treated with CIP, we conducted a retrospective cohort study. CIP non-susceptibility according to revised CIP breakpoint was not associated with recurrence of UTIs. Considering that selection of the initial empirical antibiotics could affect the clinical outcomes, we also analyzed patients who treated with CIP as an empirical antibiotic. In this subgroup analysis, CIP non-susceptibility was not associated with recurrence of UTIs within 4 weeks and 90 days. One interesting point was that time to defervescence was longer in CIP-susceptible group than CIP-non-susceptible group. This means that statistically significant differences in time to defervescence did not correlate with other clinical outcomes.

There are possible explanations for reasons why CIP non-susceptibility according to the revised CLSI did not affect primary outcomes. First, the prognosis of UTIs caused by Enterobacteriaceae was better than that of other site of infections as mentioned above. Concentrations of quinolone in urine are generally high with a major renal route of elimination, and the flushing mechanism of the bladder expels pathogens. In case of the quinolone indicated for UTIs, CIP $(30 \sim 50 \%)$ has significant renal excretion. The post antibiotic effect of quinolone also contributes to its therapeutic efficacy [17,18]. Therefore, lower MIC within non-susceptible range might not be a great concern in UTI patients. Second, less critically ill patients with relatively low CWIs and pitt bacteremia scores were included. Since patients with bacteremic UTIs due to Enterobacteriaceae, which were sensitive to CIP according to previous CLSI breakpoint, were included, it is likely that severely ill patients who had been repeatedly exposed to broad-spectrum antibiotics and hospital environment were excluded from the study. Third, when broth micro-dilution (BMD) method is used, it can reveal discrepancy in susceptibility results between the tested methods [19]. In other words, even if the CIP MIC by the automated antimicrobial susceptibility test (AST) method showed non-susceptible, there was a possibility that the CIP MIC would have been less than $0.5 \mu \mathrm{g} / \mathrm{mL}$ by the BMD method. The difference in clinical outcomes according to MIC by BMD method should be studied.

The currently revised CIP breakpoint for Enterobacteriaceae is based on PK-PD attainment analyses, which may not accurately predict clinical outcomes for all infections. This value is also not based on the mechanism of acquiring quinolone resistance genes. Quinolone resistance is principally caused by spontaneous mutation in the quinolone resistance determining region (QRDR). Multistep mutations of QRDR were responsible for high level resistance to quinolone [20,21]. However, the acquisition of plasmid-mediated quinolone resistance (PMQR) genes has been increasingly reported in Enterobacteriaceae species in most parts of the world [22,23]. The co-existence of mutations in QRDR and 
PMQR genes could occur, and the accumulation of these different mutations was associated with high level quinolone resistance [22-29]. There were also several reports that Enterobacteriaceae isolates, which are susceptible to quinolone, also had carried mutations in QRDR or PMQR genes [14,22,28-30]. There was a report that this led to them showing no clinical response when CIP resistance was acquired by the quinolone resistance gene, even when CIP MIC was about $0.25-0.5 \mu \mathrm{g} / \mathrm{mL}$ [30]. Clinicians should select appropriate antibiotics based on not only the results of AST but also the clinical response [14-16,31]. Further research should be performed on the association between acquiring quinolone resistance gene with CIP MIC and clinical outcomes.

Our study has several limitations. This is a retrospective observational study, and data collection was limited because data were gathered for only 10 years. Patients with recurrent UTIs might have sought treatment at another medical center or hospital, therefore some patients with recurrent UTIs may not have been included. Decision for choosing empirical antibiotics was made freely by physicians, resulting in major bias. The number of enrolled patients was small and showed low CWIs and Pitt bacteremia score. Our results cannot be applied to more severe UTIs presented with septic shock or cases with high risk of antibiotic resistance. Additionally, CIP MIC was measured by automated methods not by BMD method. As mentioned above, the MIC value by BMD may be different from the value by automated AST. However, our study has clinical significance reflecting real-world data where automated methods are usually used for AST.

\section{Method}

\subsection{Study Designs and Patients}

A retrospective cohort study was conducted from 2009 to 2018 in a single center, a 1950-bed tertiary care referral hospital in Seoul, South Korea. All adults patients (age > 18) diagnosed with bacteremic UTIs caused by Enterobacteriaceae considered susceptible to CIP by the prior breakpoints (CIP MIC $\leq 1 \mu \mathrm{g} / \mathrm{mL}$ ) before 2018 CLSI revision were identified. Patients receiving CIP as a definitive treatment were included. Patients receiving more than two antibiotics as definitive treatment were excluded. Included patients were divided into CIP-susceptible (MIC $\leq 0.25 \mu \mathrm{g} / \mathrm{mL}$ ) and CIP-non-susceptible groups (MIC $=0.5$ or $1 \mu \mathrm{g} / \mathrm{mL}$ ), according to the revised CLSI guideline in 2018.

\subsection{Antimicrobial Susceptibility Testing}

Species identification for all included patients was performed using the standard VITEK 2 identification card (bioMérieux Inc., Marcyl'Etoile, France) according to the CLSI susceptibility interpretative criteria [10].

\subsection{Clinical Evaluation and Outcomes}

Data were collected from electronic medical records. The following information were included: age, sex, the presence of underlying diseases or comorbid conditions, identified pathogens, types of antibiotics empirically administered, and treatment duration of antibiotics. The severity of underlying disease was estimated using CWIs. The severity of illness in bacteremia was assessed using the Pitt bacteremia score, which has been validated in several previous studies [32]. A history of urologic functional or structural abnormalities was collected as risk factors for recurrent UTIs, for example, neurogenic bladder, urinary incontinence, urolithiasis, kidney transplantation, or the presence of an indwelling foreign bodies (e.g., catheter, double J) [1]. Primary endpoints were recurrence of UTIs within 4 weeks and 90 days after administration of first antibiotics dose. Secondary endpoint included length of hospital stay from first antibiotics administration until discharge, allcause mortality, prolonged bacteremia more than 1 week, and time to defervescence reflecting short-term clinical response. Patients who received CIP as an empirical antibiotics were analyzed to determine the primary endpoint as a subgroup analysis. 


\subsection{Definitions}

UTIs were defined as a clinical syndrome characterized by costovertebral angle tenderness, fever, dysuric symptoms, pyuria, or bacteriuria [15]. Antimicrobial treatments were classified as empirical and definitive. Empirical treatment was defined as the antibiotics administrated before the final blood culture report, while definitive treatment was defined as the adjusted antibiotics based on the results of AST of blood culture isolates. Recurrence was defined as the presence of any symptoms or laboratory evidence of UTIs after discontinuation of antibiotic treatment.

\subsection{Statistical Analysis}

Differences between the CIP-susceptible and CIP-non-susceptible groups were compared using a Mann-Whitney $U$ test for continuous variables and a chi-square test for categorical variables. The Cox proportional hazard model was used to examine the association of the CIP susceptibility with recurrences within 4 weeks and 90 days by adjusting for potential confounding factors. Variables with statistical significance in the univariate analysis or potential confounders were entered into the multivariable analysis. All $p$-values were two-tailed, and those $<0.05$ were considered statistically significant. IBM SPSS Statistics version 25.0 for Windows (IBM, Armonk, NY, USA) was used for all statistical analyses. The present study was approved by Institutional review board of Samsung medical center. Informed consent was waived since the electronic medical record was reviewed retrospectively with de-personalized identification number.

\section{Conclusions}

In a retrospective cohort study of UTI patients with Enterobactericeae bacteremia treated with CIP, increased CIP MIC was not associated with relapse within 4 weeks and recurrence within 90 days. This study suggests, even if the MIC of CIP is slightly elevated $(0.5-1 \mathrm{~g} / \mathrm{mL})$ in Enterobacteriaciae, it could be possible to continue to use CIP in cases of bacteremic UTIs when patients show clinical response. However, our data cannot be extrapolated to patients with septic shock or severe infection other than UTIs.

Supplementary Materials: The following are available online at https:/ / www.mdpi.com/article/10 .3390 /antibiotics10040469/s1, Table S1: Univariate analysis of association between characteristics of patients and recurrence of urinary tract infections within 4 weeks; Table S2: Univariate analysis of association between characteristics of patients and recurrence of urinary tract infections within 90 days; Table S3: Baseline characteristics in the subgroup of patients with Enterobacteriaceae bacteremic urinary tract infections treated with ciprofloxacin as an empirical as well as a definite treatment; Table S4: Comparison of clinical outcomes in the subgroup of patients with Enterobacteriaceae bacteremic urinary tract infections treated with ciprofloxacin as an empirical as well as a definite treatment; Table S5: Univariate and multivariate analysis of association between characteristics of patients and recurrence of urinary tract infections within 4 weeks on patients treated with ciprofloxacin as an empirical as well as a definite treatment; Table S6: Univariate and multivariate analysis of association between characteristics of patients and recurrence of urinary tract infections within 90 days on patients treated with ciprofloxacin as an empirical as well as a definite treatment.

Author Contributions: Conceptualization, S.Y.C., C.-I.K., D.R.C., and K.R.P.; Investigation, G.E.P. and J.-H.K.; Methodology, G.E.P., J.-H.K., H.J.H., J.Y.B. and K.S.K.; Supervision, G.E.P. and K.R.P.; Writing—original draft, G.E.P.; Writing—review and editing, J.-H.K., S.Y.C., H.J.H., C.-I.K., D.R.C. and K.R.P. All authors have read and agreed to the publication of this manuscript.

Funding: This research received no external funding.

Institutional Review Board Statement: This study was approved by the Institutional Review Board (IRB) of Samsung medical center (IRB No. \#2021-02-016-001). The study was performed in accordance with the guidelines followed as per the Declaration of Helsinki.

Informed Consent Statement: Informed consent was waived since the electronic medical record was reviewed retrospectively with de-personalized identification number. 
Data Availability Statement: The data presented in this study are openly available in MDPI.

Conflicts of Interest: The authors declare no conflict of interest.

\section{References}

1. Hooton, T.M. Uncomplicated Urinary Tract Infection. N. Engl. J. Med. 2012, 366, 1028-1037. [CrossRef] [PubMed]

2. Walker, E.; Lyman, A.; Gupta, K.; Mahoney, M.V.; Snyder, G.M.; Hirsch, E.B. Clinical Management of an Increasing Threat: Outpatient Urinary Tract Infections Due to Multidrug-Resistant Uropathogens. Clin. Infect. Dis. Off. Publ. Infect. Dis. Soc. Am. 2016, 63, 960-965. [CrossRef]

3. Foxman, B. Epidemiology of urinary tract infections: Incidence, morbidity, and economic costs. Am. J. Med. 2002, 5s-13s. [CrossRef]

4. Wayne, P. CLSI. Fluoroquinolone Breakpoints for Enterobacteriaceae and Pseudomonas Aeruginosa. 1st ed. CLSI Ratinale Document MR02. Available online: www.clsi.org (accessed on 20 February 2021).

5. Lettieri, J.T.; Rogge, M.C.; Kaiser, L.; Echols, R.M.; Heller, A.H. Pharmacokinetic profiles of ciprofloxacin after single intravenous and oral doses. Antimicrob. Agents Chemother. 1992, 36, 993-996. [CrossRef]

6. Kim, E.S.; Hooper, D.C. Clinical Importance and Epidemiology of Quinolone Resistance. Infect. Chemother. 2014, 46, 226-238. [CrossRef] [PubMed]

7. Hooper, D.C.; Jacoby, G.A. Mechanisms of drug resistance: Quinolone resistance. Ann. N. Y. Acad. Sci. 2015, $1354,12-31$. [CrossRef] [PubMed]

8. Performance Standards for Antimicrobial Susceptibility Testing, 28th ed.; CLSI Supplement M100; The Clinical Laboratory Standards Institute: Wayne, PA, USA, 2018.

9. Humphries, R.M.; Abbott, A.N.; Hindler, J.A. Understanding and Addressing CLSI Breakpoint Revisions: A Primer for Clinical Laboratories. J. Clin. Microbiol. 2019, 57. [CrossRef]

10. Performance Standards for Antimicrobial Susceptibility Testing, 29th ed; CLSI Supplement M100; The Clinical Laboratory Standards Institute: Wayne, PA, USA, 2019.

11. Forrest, A.; Nix, D.E.; Ballow, C.H.; Goss, T.F.; Birmingham, M.C.; Schentag, J.J. Pharmacodynamics of intravenous ciprofloxacin in seriously ill patients. Antimicrob. Agents Chemother. 1993, 37, 1073-1081. [CrossRef] [PubMed]

12. Drusano, G.L.; Preston, S.L.; Fowler, C.; Corrado, M.; Weisinger, B.; Kahn, J. Relationship between fluoroquinolone area under the curve: Minimum inhibitory concentration ratio and the probability of eradication of the infecting pathogen, in patients with nosocomial pneumonia. J. Infect. Dis. 2004, 189, 1590-1597. [CrossRef]

13. Ebbing, L.; Metlay, J.P.; Bilker, W.B.; Edelstein, P.H.; Fishman, N.O. Association between Fluoroquinolone Resistance and Mortality in Escherichia coli and Klebsiella pneumoniae Infections: The Role of Inadequate Empirical Antimicrobial Therapy. Clin. Infect. Dis. 2005, 41, 923-929. [CrossRef]

14. Peralta, G.; Sánchez, M.B.; Garrido, J.C.; De Benito, I.; Cano, M.E.; Martínez-Martínez, L.; Roiz, M.P. Impact of antibiotic resistance and of adequate empirical antibiotic treatment in the prognosis of patients with Escherichia coli bacteraemia. J. Antimicrob. Chemother. 2007, 60, 855-863. [CrossRef]

15. Lee, S.S.; Kim, Y.; Chung, D.R. Impact of discordant empirical therapy on outcome of community-acquired bacteremic acute pyelonephritis. J. Infect. 2011, 62, 159-164. [CrossRef]

16. Jeon, J.H.; Kim, K.; Han, W.D.; Song, S.H.; Park, K.U.; Rhee, J.E.; Song, K.H.; Park, W.B.; Kim, E.S.; Park, S.W.; et al. Empirical use of ciprofloxacin for acute uncomplicated pyelonephritis caused by Escherichia coli in communities where the prevalence of fluoroquinolone resistance is high. Antimicrob. Agents Chemother. 2012, 56, 3043-3046. [CrossRef]

17. Gupta, K.; Hooton, T.M.; Stamm, W.E. Increasing antimicrobial resistance and the management of uncomplicated communityacquired urinary tract infections. Ann. Intern. Med. 2001, 135, 41-50. [CrossRef]

18. Chen, Y.H.; Ko, W.C.; Hsueh, P.R. The role of fluoroquinolones in the management of urinary tract infections in areas with high rates of fluoroquinolone-resistant uropathogens. Eur. J. Clin. Microbiol. Infect. Dis. 2012, 31, 1699-1704. [CrossRef]

19. Zhou, M.; Wang, Y.; Liu, C.; Kudinha, T.; Liu, X.; Luo, Y.; Yang, Q.; Sun, H.; Hu, J.; Xu, Y.C. Comparison of five commonly used automated susceptibility testing methods for accuracy in the China Antimicrobial Resistance Surveillance System (CARSS) hospitals. Infect. Drug Resist. 2018, 11, 1347-1358. [CrossRef] [PubMed]

20. Gales, A.C.; Gordon, K.A.; Wilke, W.W.; Pfaller, M.A.; Jones, R.N. Occurrence of single-point gyrA mutations among ciprofloxacinsusceptible Escherichia coli isolates causing urinary tract infections in Latin America. Diagn. Microbiol. Infect. Dis. 2000, 36, 61-64. [CrossRef]

21. Pourahmad Jaktaji, R.; Mohiti, E. Study of Mutations in the DNA gyrase gyrA Gene of Escherichia coli. Iran J. Pharm Res. 2010, 9, 43-48.

22. Kim, H.B.; Park, C.H.; Kim, C.J.; Kim, E.C.; Jacoby, G.A.; Hooper, D.C. Prevalence of plasmid-mediated quinolone resistance determinants over a 9-year period. Antimicrob. Agents Chemother. 2009, 53, 639-645. [CrossRef]

23. Yang, H.Y.; Nam, Y.S.; Lee, H.J. Prevalence of plasmid-mediated quinolone resistance genes among ciprofloxacin-nonsusceptible Escherichia coli and Klebsiella pneumoniae isolated from blood cultures in Korea. Can. J. Infect. Dis. Med Microbiol. 2014, 25, 163-169. [CrossRef] 
24. Kotb, D.N.; Mahdy, W.K.; Mahmoud, M.S.; Khairy, R.M.M. Impact of co-existence of PMQR genes and QRDR mutations on fluoroquinolones resistance in Enterobacteriaceae strains isolated from community and hospital acquired UTIs. BMC Infect. Dis. 2019, 19, 979. [CrossRef] [PubMed]

25. Ranjbar, R.; Tolon, S.S.; Sami, M.; Golmohammadi, R. Detection of Plasmid-Mediated qnr Genes Among the Clinical QuinoloneResistant Escherichia coli Strains Isolated in Tehran, Iran. Open Microbiol. J. 2018, 12, 248-253. [CrossRef] [PubMed]

26. Ho, H.J.; Tan, M.X.; Chen, M.I.; Tan, T.Y.; Koo, S.H.; Koong, A.Y.L.; Ng, L.P.; Hu, P.L.; Tan, K.T.; Moey, P.K.S.; et al. Interaction between Antibiotic Resistance, Resistance Genes, and Treatment Response for Urinary Tract Infections in Primary Care. J. Clin. Microbiol. 2019, 57. [CrossRef] [PubMed]

27. Salah, F.D.; Soubeiga, S.T.; Ouattara, A.K.; Sadji, A.Y.; Metuor-Dabire, A.; Obiri-Yeboah, D.; Banla-Kere, A.; Karou, S.; Simpore, J. Distribution of quinolone resistance gene (qnr) in ESBL-producing Escherichia coli and Klebsiella spp. in Lomé, Togo. Antimicrob. Resist. Infect. Control. 2019, 8, 104. [CrossRef]

28. Esmaeel, N.E.; Gerges, M.A.; Hosny, T.A.; Ali, A.R.; Gebriel, M.G. Detection of Chromosomal and Plasmid-Mediated Quinolone Resistance Among Escherichia coli Isolated from Urinary Tract Infection Cases; Zagazig University Hospitals, Egypt. Infect. Drug Resist. 2020, 13, 413-421. [CrossRef]

29. Komp Lindgren, P.; Karlsson, A.; Hughes, D. Mutation rate and evolution of fluoroquinolone resistance in Escherichia coli isolates from patients with urinary tract infections. Antimicrob. Agents Chemother. 2003, 47, 3222-3232. [CrossRef]

30. Seok, H.; Cha, M.K.; Kang, C.I.; Cho, S.Y.; Kim, S.H.; Ha, Y.E.; Chung, D.R.; Peck, K.R.; Song, J.H. Failure of Ciprofloxacin Therapy in the Treatment of Community-Acquired Acute Pyelonephritis caused by In-Vitro Susceptible Escherichia coli Strain Producing CTX-Type Extended-Spectrum $\beta$-Lactamase. Infect. Chemother. 2018, 50, 357-361. [CrossRef]

31. Kang, C.I.; Kim, S.H.; Park, W.B.; Lee, K.D.; Kim, H.B.; Kim, E.C.; Oh, M.D.; Choe, K.W. Bloodstream infections due to extendedspectrum beta-lactamase-producing Escherichia coli and Klebsiella pneumoniae: Risk factors for mortality and treatment outcome, with special emphasis on antimicrobial therapy. Antimicrob. Agents Chemother. 2004, 48, 4574-4581. [CrossRef]

32. Rhee, J.Y.; Kwon, K.T.; Ki, H.K.; Shin, S.Y.; Jung, D.S.; Chung, D.R.; Ha, B.C.; Peck, K.R.; Song, J.H. Scoring systems for prediction of mortality in patients with intensive care unit-acquired sepsis: A comparison of the Pitt bacteremia score and the Acute Physiology and Chronic Health Evaluation II scoring systems. Shock 2009, 31, 146-150. [CrossRef] 